\title{
More pain, more gain: blocking the opioid system boosts adaptive cognitive control
}

\author{
Henk van Steenbergen ${ }^{1,2}$, Daniel H. Weissman ${ }^{3}$, Dan J. Stein ${ }^{4}$, Susan Malcolm-Smith ${ }^{5}$, \\ \& Jack van Honk ${ }^{4,6,7}$
}

\section{Manuscript accepted for publication in Psychoneuroendocrinology}

\author{
Corresponding author: \\ Henk van Steenbergen \\ Wassenaarseweg 52 \\ 2333 AK Leiden \\ The Netherlands \\ Phone number: $+31(0) 715273655$ \\ Fax number: +31 (0)71 5273783 \\ E-mail address: HvanSteenbergen@ fsw.leidenuniv.nl \\ Lab website: www.henkvansteenbergen.com
}

\section{Keywords:}

Cognitive control, mu-opioid system, anterior cingulate, post-error slowing, conflict adaptation

\section{Author contributions:}

J. van Honk and H. van Steenbergen developed the study concept. All authors contributed to the study design. H. van Steenbergen performed the data analysis and interpretation and drafted the manuscript. All other authors provided critical revisions. All authors approved the final version of the manuscript for submission

Task and data are available online: https://osf.io/ct3hx/ 


\begin{abstract}
The ability to adaptively increase cognitive control in response to cognitive challenges is crucial for goal-directed behavior. Recent findings suggest that aversive arousal triggers adaptive increases of control, but the neurochemical mechanisms underlying these effects remain unclear. Given the known contributions of the opioid system to hedonic states, we investigated whether blocking this system increases adaptive control modulations. To do so, we conducted a double-blind, placebo-controlled psychopharmacological study ( $\mathrm{n}=52$ females) involving a Stroop-like task. Specifically, we assessed the effect of naltrexone, an opioid blocker most selective to the mu-opioid system, on two measures of adaptive control that are thought to depend differentially on aversive arousal: post-error slowing and conflict adaptation. Consistent with our hypothesis, relative to placebo, naltrexone increased post-error slowing without influencing conflict adaptation. This finding not only supports the view that aversive arousal triggers adaptive control but also reveals a novel role for the opioid system in modulating such effects.
\end{abstract}




\section{Introduction}

Adaptively increasing cognitive control in response to cognitive challenges is a key component of goal-directed behavior. Recently, it has been suggested that aversive arousal associated with cognitive challenges triggers such adaptive control (Dreisbach and Fischer, 2012; Inzlicht et al., 2015; van Steenbergen, 2015). Consistent with this view, in Stroop-like tasks affective processes contribute to behavioral adjustments that reflect adaptive control following events that trigger aversive arousal, such as errors (Bartholow et al., 2012) and high-conflict incongruent trials (van Steenbergen et al., 2009). Further, in line with mood-congruency theories (van Steenbergen, 2015), these behavioral adjustments are attenuated by the induction of hedonic states (van Steenbergen et al., 2015, 2010) and by the intake of drugs that have anxiolytic or analgesic effects (Bartholow et al., 2012; Randles et al., 2016; Ridderinkhof et al., 2002). Thus, rather than positing affect is the enemy of cognitive control (Metcalfe and Mischel, 1999), an emerging view is that at least one form of affect - phasic increases of aversive arousal - triggers cognitive control (Dreisbach and Fischer, 2012; Inzlicht et al., 2015; Inzlicht and Legault, 2014; van Steenbergen, 2015).

Although prior work has revealed the neural structures that are responsible for adaptive control (Botvinick et al., 2001) and the physiological effects of aversive conflict processing (Lindström et al., 2013; van Steenbergen and Band, 2013), little is known about the neurochemical mechanisms that underlie adaptive control. Given the known role of mu-opioids in producing the hedonic dimension of affective states (Kringelbach and Berridge, 2009; Leknes and Tracey, 2008), we recently hypothesized that the mu-opioid system reduces the phasic aversive arousal response to cognitive challenges and thereby reduces control-triggered behavioral adjustments (van Steenbergen, 2015; van Steenbergen et al., 2015). More specifically, we proposed that mu-opioids reduce behavioral adjustments via their influence on the anterior cingulate cortex, a brain region that (a) supports aversive processing and cognitive control (Braem et al., 2017; Shackman et al., 2011; Shenhav et al., 2016) and (b) has a high expression of muopioid receptors (Bush et al., 2000; Zubieta et al., 2003). The actual influence of mu-opioids on cognitive 
control remains unclear, however, because, until recently, psychopharmacological studies of endogenous mu-opioids were typically limited to pleasure and pain processing. Although pleasure and pain may share psychological and neural features with cognitive control (Inzlicht and Legault, 2014), they also differ from cognitive control in important ways.

We therefore employed a psychopharmacological manipulation to test for the first time whether opioid transmission influences adaptive control in a Stroop-like task. Our study capitalized on two wellknown behavioral adjustments that are generally considered to be hallmark manifestations of adaptive control (Botvinick et al., 2001; cf. Figure 1): (1) cautious responding following error trials, as measured by post-error slowing (i.e., slower responses after errors than after correct trials; Rabbitt, 1966); and (2) increased attentional focusing following correct incongruent trials, as measured by conflict adaptation (i.e., a smaller congruency effect following incongruent relative to congruent trials; Gratton et al., 1992).

As described earlier, we have hypothesized that opioids act on mu receptors to blunt phasic demand-related aversive arousal, thereby reducing control-triggered behavioral adjustments (van Steenbergen, 2015; van Steenbergen et al., 2015). We therefore predicted that, in comparison to placebo, antagonizing mu-opioid receptors would increase such adjustments. Given that errors are thought to elicit stronger aversive arousal than conflict in correctly-performed incongruent trials (Hajcak and Foti, 2008; Inzlicht et al., 2015), we further predicted that antagonizing mu-opioid receptors would increase post-error slowing but not necessarily conflict adaptation. We tested these hypotheses using a double-blind, placebocontrolled experimental design wherein one group of participants received naltrexone, an opioid blocker that is most selective to the mu-opioid system, while a second group received a placebo.

\section{Materials and Method}

\subsection{Participants}

The data from the Stroop-like task described here were collected during a study at Cape Town University that investigated the effects of opioids on social behavior. This study involved 52 female participants (age: $18-27, M=20.3$ years). All participants were of South African nationality, Caucasian, 
and right handed. Exclusion criteria included a history of psychopathology, use of alcohol or painkillers in the last 24 hours, and use of psychotropic medication. The Human Research Ethics Committee (HREC) at the University of Cape Town approved the study protocol. Participants gave informed written consent prior to the study and received financial compensation afterwards.

We excluded eight participants following completion of the study. One did not complete the task, four performed at chance levels of accuracy, one had response omissions on more than $15 \%$ of the trials, one never made errors, and one reported severe symptoms of depression $($ BDI score $=31$ ). Thus, 44 participants were available for data analysis. Four additional participants were excluded because their data were characterized by extreme outliers (i.e., more than 3 interquartile ranges below/above the 25 th/75th percentile) on one or more behavioral scores (see below). The final data set therefore included 40 participants (18 in the naltrexone group, 22 in the placebo group).

\subsection{Procedure}

After participants signed the informed consent document, we randomly assigned them, in a double-blind fashion, to one of two groups. Participants in the experimental group $(\mathrm{N}=26)$ orally received $50 \mathrm{mg}$ of the opioid antagonist naltrexone. Participants in the control group $(\mathrm{N}=26)$ orally received a placebo. Participants began the Stroop-like task 76 minutes after the administration of either naltrexone or placebo and stopped six minutes later. These temporal parameters were chosen to coincide with the central effects of naltrexone (Lee et al., 1988).

To test our hypothesis, we employed a Stroop-like task (Schmidt and Weissman, 2014). In each 2 s trial (see Figure 1), four frames were presented consecutively: a distractor (133 ms), a blank screen (33 $\mathrm{ms})$, a target (133 ms), and a second blank screen $(1700 \mathrm{~ms})$ during which the response was recorded. The distracter consisted of three identical direction words ('Left', 'Right', 'Up', or 'Down'; 48-point Courier New font) stacked vertically at the center of the display. The target was a single word at the center of the display ('Left, 'Right, 'Up' or 'Down'; 77-point Courier New font). We instructed participants to press a key on a computer keyboard as quickly and as accurately as possible to identify the target. In particular, 
we instructed participants to press $\mathrm{F}$ (left middle finger), $\mathrm{G}$ (left index finger), $\mathrm{J}$ (right middle finger) or $\mathrm{N}$ (right index finger), respectively, to indicate that the target was 'Left, 'Right, 'Up' or 'Down'. The word 'Error' (60-point Courier new font) appeared for $200 \mathrm{~ms}$ following incorrect responses and response omissions (i.e., the absence of a response to the target within $1500 \mathrm{~ms}$ of target onset). The task was presented on a 15-inch monitor $(1280$ x 1024 px @ $60 \mathrm{~Hz})$ via E-Prime software. All stimuli appeared in white on a black background.

It is important to mention that we designed our task to avoid feature integration and contingency learning confounds whose presence complicates the ability to interpret conflict adaptation as an index of cognitive control (Schmidt, 2013). To avoid feature integration confounds, which occur when stimuli or responses repeat in consecutive trials, we divided our 4-alternative forced choice (4-AFC) task into a pair of 2-AFC tasks (Schmidt and Weissman, 2014). The Left-Right task consisted of four distracter-target pairs made of the words left and/or right (congruent: Left-Left, Right-Right; incongruent: Left-Right, Right-Left). The Up-Down task consisted of four distracter-target pairs made of the words up and/or down (congruent: Up-Up, Down-Down; incongruent: Up-Down, Down-Up). To avoid stimulus and response repetitions in consecutive trials, we presented distracter-target pairs from the Left-Right task in odd trials of each block and distracter-target pairs from the Up-Down task in even trials. To avoid contingency learning confounds, we presented each distracter-target pair equally often in every block of trials (Schmidt and Weissman, 2014).

Participants performed a single block of 24 practice trials (about 48 seconds) and two blocks of 96 test trials (about 3 minutes and 12 seconds each). Each block was followed by a self-paced break. Selfreported mood was assessed at the end of the study using a computerized version of the Positive Affect Negative Affect Scale (Watson et al., 1988).

\subsection{Behavioral analyses}

To assess adaptive control following errors, we calculated post-error slowing using the optimized method described by Dutilh and colleagues (2012). This method yields a measure that is not confounded 
by global fluctuations in task performance over time (e.g., due to motivation or attention). More specifically, we isolated triplets of trials in which errors were preceded and followed by a correct trial. Individual mean post-error slowing scores were then calculated as follows: PES = RT post-error minus RT pre-error. Post-error accuracy was calculated by comparing mean accuracy after errors to mean accuracy after correct trials using trials from the entire data set: PEA $=$ Accuracy post-error minus Accuracy postcorrect.

To assess conflict adaptation (see van Steenbergen et al., 2010), we subtracted the congruency effect following incongruent trials from the congruency effect following congruent trials (separately for mean RT and mean accuracy). We can express this mathematically with the following quantity wherein small letters indicate the congruency of the previous trial and capital letters indicate the congruency of the current trial: $(\mathrm{cI}-\mathrm{cC})-(\mathrm{iI}-\mathrm{iC})$. For the RT analysis, we excluded the first trial of each block, incorrect trials, and trials that followed incorrect trials. Based on the remaining data, we then excluded outliers (i.e., correctly-performed trials with RTs greater than 2 SDs from their condition-specific mean, calculated for each participant separately). For the accuracy analysis, we excluded the same trials with the exception of incorrect trials and RT outliers, because this analysis focused solely on whether participants made the correct or incorrect response. For completeness, we also report mean congruency effects and grand average scores (see Table 1).

To investigate whether naltrexone increases post-error slowing, conflict adaptation, or negative mood, we submitted the calculated behavioral measures of adaptive control (i.e., post-error slowing and conflict adaptation, see above) and self-reported mood scores to separate ANOVAs with a single betweenparticipants factor: Group (placebo, naltrexone). Tests for the intercept parameter were used to determine whether the typical congruency, conflict adaptation, and post-error slowing effects were present across the entire sample. A significance level of $\mathrm{p}<.05$ was adopted for all statistical tests. In accordance with the journal's guidelines, we also report all non-significant findings with $.05<\mathrm{p}<.249$ (see Table 1 for descriptive statistics). 


\section{Results}

When collapsing the data across groups, we observed the expected congruency effect and behavioral adjustments. First, the congruency effect was significant in both mean RT, F(1,38)=190.81, $\mathrm{p}<.001, \mathrm{MSE}=1952.1, \eta_{p}^{2}=.834$, and mean accuracy, $\mathrm{F}(1,38)=29.22, \mathrm{p}<.001, \mathrm{MSE}=0.001, \eta_{p}^{2}=.435$. Second, in mean RT, we observed both post-error slowing, $F(1,38)=15.04$, p<.001, MSE $=14790.3$, $\eta_{p}^{2}=.284$, and conflict adaptation, $\mathrm{F}(1,38)=15.53, \mathrm{p}<.001, \mathrm{MSE}=1891.0, \eta_{p}^{2}=.290$. Third, in mean accuracy, we observed greater accuracy after errors than after correct responses, $\mathrm{F}(1,38)=28.52$, $\mathrm{p}<.001$, MSE $=0.001, \eta_{p}^{2}=.429$. This finding suggests that the post-error slowing we observed reflected an attempt to trade speed for accuracy after errors, rather than a reduction of attention to the task. Fourth, although we did not observe conflict adaptation in mean accuracy, $\mathrm{F}(1,38)=1.24, \mathrm{p}=.272$, MSE $=0.004, \eta_{p}^{2}=.032$, the absence of this effect indicates that the conflict adaptation effect we observed in mean RT did not index a speed-accuracy tradeoff.

Two additional findings confirmed our main hypothesis. First, post-error slowing was greater in the naltrexone group than in the placebo group, $\mathrm{F}(1,38)=8.88, \mathrm{p}=.005$, MSE $=14790.3, \eta_{p}^{2}=.189$ (Figure 2). We did not observe an analogous group difference for post-error accuracy, $F(1,38)=0.01, p=.930$, MSE $=0.001, \eta_{p}^{2}=.0002$, likely because errors followed other errors very infrequently, leading to a floor effect for this measure (see Table 1). Second, conflict adaptation did not differ between the groups in either mean RT, $\mathrm{F}(1,38)=0.50, \mathrm{p}=.485, \mathrm{MSE}=1891.0, \eta_{p}^{2}=.013$, or mean accuracy, $\mathrm{F}(1,38)=1.81, \mathrm{p}=.187$, $\mathrm{MSE}=0.004, \eta_{p}^{2}=.045$.

Finally, we note that analyses of self-reported hedonic tone, as assessed by the PANAS, revealed no significant effects of naltrexone. First, there were no significant effects of naltrexone on positive affect, $\mathrm{F}(1,38)=0.03, \mathrm{p}=.855, \mathrm{MSE}=0.517, \eta_{p}^{2}=.001$. Second, there were no significant effects of naltrexone on negative affect: $\mathrm{F}(1,38)=0.03, \mathrm{p}=.860, \mathrm{MSE}=0.192, \eta_{p}^{2}=.001$.

\section{Discussion}


The present findings provide novel support for the view that phasic increases of aversive arousal trigger adaptive increases in cognitive control. More specifically, our findings indicate that blocking opioid receptors with naltrexone, which should prevent the opioid system from blunting aversive arousal associated with errors, increases post-error slowing. In isolation, this effect could be attributed to an orienting response that draws attention away from a task (Notebaert et al., 2009). However, this was not the case in the present study because, across groups, post-error slowing was accompanied by higher (rather than lower) post-error accuracy. Our findings therefore indicate that blocking central opioid function boosts adaptive control following errors (cf. Murphy et al., 2016). To our knowledge, this is the first demonstration that the opioid system plays a causal role in modulating adaptive control.

In contrast, we did not observe an overall effect of our pharmacological intervention on a second measure of adaptive control known as conflict adaptation. This finding is consistent with earlier work suggesting that correctly-performed incongruent trials evoke relatively weak phasic aversive arousal responses in comparison to errors (Hajcak and Foti, 2008; Inzlicht et al., 2015). This outcome might appear to contradict prior findings showing that the hedonic tone of induced mood influences conflict adaptation (Kuhbandner and Zehetleitner, 2011; Schuch and Koch, 2015; van Steenbergen et al., 2012, 2010). This contradiction is more apparent than real, however, as naltrexone did not significantly alter tonic affect (as assessed by the PANAS) in the present study. In sum, our findings indicate that naltrexone increases post-error slowing but not conflict adaptation, consistent with the view that errors induce larger phasic increases of aversive arousal than correctly-performed incongruent trials (Inzlicht et al., 2015).

The naltrexone-induced improvement in adaptive control that we have observed may reflect a modulation of the fronto-parietal network that underlies cognitive control (Cocchi et al., 2013). Within this neural network, the anterior cingulate cortex (ACC) is a critical hub that is thought to monitor the need for heightened cognitive control (e.g., following errors) (Botvinick et al., 2001; Shenhav et al., 2016; Spunt et al., 2012). Further, brain activity in this region is modulated by the hedonic state of the individual (van Steenbergen et al., 2015), likely because this region contains a high number of mu-opioid receptors 
(Bush et al., 2000; Zubieta et al., 2003). Future research might therefore investigate whether the effects of naltrexone that we have observed reflect, at least partly, the blocking of mu-opioid receptors in the ACC.

Mu-opioids also influence other neural mechanisms. First, they influence other brain regions, such as the basal ganglia, which indirectly influence the fronto-parietal control network via corticostriatal interactions (van Steenbergen et al., 2015). Second, they interact with neurotransmitters such as dopamine and norepinephrine (Jocham and Ullsperger, 2009; Ullsperger et al., 2014), which may exert affective influences on cognitive control and decision making that are related to motivation and arousal (Barbano and Cador, 2007; Notebaert and Braem, 2016; van Steenbergen, 2015). Future studies might therefore also investigate whether the effects we have observed index the effects of opioids on these brain mechanisms. More broadly, the influence of opioids on various brain mechanisms might be partly responsible for modulations of cognitive control that are associated with ingesting substances that increase endogenous opioids, such as alcohol (Bartholow et al., 2012; Ridderinkhof et al., 2002). Finally, an interesting topic for future research concerns the link between opioids and task-switching, another important aspect of cognitive control which has been shown to be modulated by positive affect (for reviews, see Aarts et al., 2012; Chiew and Braver, 2011).

Finally, it is important to mention two limitations of the present study. First, although naltrexone shows the highest sensitivity for the mu-opioid system (Codd et al., 1995), we cannot exclude the possibility that it also influences the kappa-opioid and perhaps the delta-opioid receptor systems, and that these systems also modulate cognitive control processes (cf. Pfabigan et al., 2015). Second, since the present study only tested females, additional studies will be required to assess whether the present findings generalize to males.

\section{Conclusions}

In sum, our findings provide the first evidence that mu-opioids play a causal role in modulating adaptive control following events that trigger high aversive arousal (i.e., errors). Our findings therefore reveal that opioids are important not only for feeling life's immediate pains and pleasures, but also for 
modulating adaptive increases in cognitive control. Future studies aimed at identifying the opioidmediated neural circuitry that underlies the effects we have observed may shed additional light on how the opioid system modulates adaptive control following aversive events. 
Van Steenbergen et al. - Opioids modulate post-error slowing

\section{Acknowledgements}

We thank Lisa Rosenberger and Kate Hamilton for their help in data acquisition. Dan Stein is funded by the South African Medical Research Council (NRF). 


\section{References}

Aarts, E., van Holstein, M., Cools, R., 2012. Striatal dopamine and the interface between motivation and cognition. Front. Psychol. 2, 163.

Barbano, M.F., Cador, M., 2007. Opioids for hedonic experience and dopamine to get ready for it. Psychopharmacology (Berl). 191, 497-506.

Bartholow, B.D., Henry, E.A., Lust, S.A., Saults, J.S., Wood, P.K., 2012. Alcohol effects on performance monitoring and adjustment: Affect modulation and impairment of evaluative cognitive control. J. Abnorm. Psychol. 121, 173-186.

Botvinick, M.M., Braver, T.S., Barch, D.M., Carter, C.S., Cohen, J.D., 2001. Conflict monitoring and cognitive control. Psychol. Rev. 108, 624-652.

Braem, S., King, J.A., Korb, F.M., Krebs, R.M., Notebaert, W., Egner, T., 2017. The role of anterior cingulate cortex in the affective evaluation of conflict. J. Cogn. Neurosci. 29, 137-149. doi:10.1162/jocn_a_01023

Bush, G., Luu, P., Posner, M.I., 2000. Cognitive and emotional influences in anterior cingulate cortex. Trends Cogn. Sci. 4, 215-222.

Chiew, K.S., Braver, T.S., 2011. Positive affect versus reward: emotional and motivational influences on cognitive control. Front. Psychol. 2, 279.

Cocchi, L., Zalesky, A., Fornito, A., Mattingley, J.B., 2013. Dynamic cooperation and competition between brain systems during cognitive control. Trends Cogn. Sci. 17, 493-501. doi:10.1016/j.tics.2013.08.006

Codd, E.E., Shank, R.P., Schupsky, J.J., Raffa, R.B., 1995. Serotonin and norepinephrine uptake inhibiting activity of centrally acting analgesics: structural determinants and role in antinociception. J. Pharmacol. Exp. Ther. 274, 1263-1270.

Dreisbach, G., Fischer, R., 2012. The role of affect and reward in the conflict-triggered adjustment of 
cognitive control. Front. Hum. Neurosci. 6, 342.

Dutilh, G., van Ravenzwaaij, D., Nieuwenhuis, S., van der Maas, H.L.J., Forstmann, B.U., Wagenmakers, E.-J., 2012. How to measure post-error slowing: a confound and a simple solution. J. Math. Psychol. $56,208-216$.

Gratton, G., Coles, M.G.H., Donchin, E., 1992. Optimizing the use of information: Strategic control of activation of responses. J. Exp. Psychol. Gen. 121, 480-506. doi:10.1037/0096-3445.121.4.480

Hajcak, G., Foti, D., 2008. Errors are aversive: Defensive motivation and the error-related negativity. Psychol. Sci. 19, 103-108. doi:10.1111/j.1467-9280.2008.02053.x

Inzlicht, M., Bartholow, B.D., Hirsh, J.B., 2015. Emotional foundations of cognitive control. Trends Cogn. Sci. 19, 1-7. doi:10.1016/j.tics.2015.01.004

Inzlicht, M., Legault, L., 2014. No pain, no gain: How distress underlies effective self-control (and unites diverse social psychological phenomena), in: Forgas, J.P., Harmon-Jones, E. (Eds.), Motivation and Its Regulation: The Control Within. Psychology Press, New York, NY, pp. 115-132. doi:10.1017/9781315795263

Jocham, G., Ullsperger, M., 2009. Neuropharmacology of performance monitoring. Neurosci. Biobehav. Rev. 33, 48-60.

Kringelbach, M.L., Berridge, K.C., 2009. Towards a functional neuroanatomy of pleasure and happiness. Trends Cogn. Sci. 13, 479-487.

Kuhbandner, C., Zehetleitner, M., 2011. Dissociable effects of valence and arousal in adaptive executive control. PLoS One 6, e29287.

Lee, M.C., Wagner Jr, H.N., Tanada, S., Frost, J.J., Bice, A.N., Dannals, R.F., 1988. Duration of occupancy of opiate receptors by naltrexone. J. Nucl. Med. 29, 1207-1211.

Leknes, S., Tracey, I., 2008. Science \& society - A common neurobiology for pain and pleasure. Nat. Rev. Neurosci. 9, 314-320. 
Lindström, B.R., Mattsson-Mårn, I.B., Golkar, A., Olsson, A., 2013. In your face: Risk of punishment enhances cognitive control and error-related activity in the corrugator supercilii muscle. PLoS One 8, e65692. doi:10.1371/journal.pone.0065692

Metcalfe, J., Mischel, W., 1999. A hot/cool-system analysis of delay of gratification: dynamics of willpower. Psychol. Rev. 106, 3-19. doi:10.1037/0033-295X.106.1.3

Murphy, P.R., van Moort, M.L., Nieuwenhuis, S., 2016. The pupillary orienting response predicts adaptive behavioral adjustment after errors. PLoS One 11, e0151763. doi:10.1371/journal.pone.0151763

Notebaert, W., Braem, S., 2016. Parsing the effect of reward on cognitive control, in: Braver, T.S. (Ed.), Motivation and Cognitive Control. Psychology Press, New York, NY, pp. 105-122.

Notebaert, W., Houtman, F., Van Opstal, F., Gevers, W., Fias, W., Verguts, T., 2009. Post-error slowing: an orienting account. Cognition 111, 275-279.

Pfabigan, D.M., Pripfl, J.J., Kroll, S.L., Sailer, U., Lamm, C., 2015. Event-related potentials in performance monitoring are influenced by the endogenous opioid system. Neuropsychologia 77, 242-252. doi:10.1016/j.neuropsychologia.2015.08.028

Rabbitt, P.M., 1966. Errors and error correction in choice-response tasks. J. Exp. Psychol. 71, 264.

Randles, D., Kam, J.W.Y., Heine, S.J., Inzlicht, M., Handy, T.C., 2016. Acetaminophen attenuates error evaluation in cortex. Soc. Cogn. Affect. Neurosci. nsw023. doi:10.1093/scan/nsw023

Ridderinkhof, K.R., de Vlugt, Y., Bramlage, A., Spaan, M., Elton, M., Snel, J., Band, G.P.H., 2002. Alcohol consumption impairs detection of performance errors in mediofrontal cortex. Science (80-. ). 298, 2209-2211.

Schmidt, J.R., 2013. Questioning conflict adaptation: proportion congruent and Gratton effects reconsidered. Psychon. Bull. Rev. 20, 615-30. doi:10.3758/s13423-012-0373-0

Schmidt, J.R., Weissman, D.H.D.D.H., 2014. Congruency sequence effects without feature integration or 
contingency learning confounds. PLoS One 9, e102337. doi:10.1371/journal.pone.0102337

Schuch, S., Koch, I., 2015. Mood states influence cognitive control: The case of conflict adaptation. Psychol. Res. 79, 759-772.

Shackman, A.J., Salomons, T. V, Slagter, H.A., Fox, A.S., Winter, J.J., Davidson, R.J., 2011. The integration of negative affect, pain and cognitive control in the cingulate cortex. Nat. Rev. Neurosci. 12, 154-167. doi:10.1038/nrn2994

Shenhav, A., Cohen, J.D., Botvinick, M.M., 2016. Dorsal anterior cingulate cortex and the value of control. Nat. Neurosci. 19, 1286-1291. doi:10.1038/nn.4382

Spunt, R.P., Lieberman, M.D., Cohen, J.R., Eisenberger, N.I., 2012. The phenomenology of error processing: The dorsal ACC response to stop-signal errors tracks reports of negative affect. J. Cogn. Neurosci. 24, 1753-1765. doi:10.1162/jocn_a_00242

Ullsperger, M., Danielmeier, C., Jocham, G., 2014. Neurophysiology of performance monitoring and adaptive behavior. Physiol. Rev. 94, 35-79. doi:10.1152/physrev.00041.2012

van Steenbergen, H., 2015. Affective modulation of cognitive control: A biobehavioral perspective. Handb. Biobehav. Approaches to Self-Regulation 31, 89-107. doi:10.1007/978-1-4939-1236-0_7

van Steenbergen, H., Band, G.P.H., 2013. Pupil dilation in the Simon task as a marker of conflict processing. Front. Hum. Neurosci. 7, 215. doi:10.3389/fnhum.2013.00215

van Steenbergen, H., Band, G.P.H., Hommel, B., 2010. In the mood for adaptation: How affect regulates conflict-driven control. Psychol. Sci. 21, 1629-1634. doi:10.1177/0956797610385951

van Steenbergen, H., Band, G.P.H., Hommel, B., 2009. Reward counteracts conflict adaptation: evidence for a role of affect in executive control. Psychol. Sci. 20, 1473-1477. doi:10.1111/j.14679280.2009.02470.x

van Steenbergen, H., Band, G.P.H., Hommel, B., Rombouts, S.A.R.B., Nieuwenhuis, S., 2015. Hedonic hotspots regulate cingulate-driven adaptation to cognitive demands. Cereb. cortex. 
doi:10.1093/cercor/bht416

van Steenbergen, H., Booij, L., Band, G.P.H., Hommel, B., van der Does, A.J.W., 2012. Affective regulation of cognitive-control adjustments in remitted depressive patients after acute tryptophan depletion. Cogn. Affect. Behav. Neurosci. 12, 280-286. doi:10.3758/s13415-011-0078-2

Watson, D., Clark, L.A., Tellegen, A., 1988. Development and validation of brief measures of positive and negative affect - the PANAS scales. J. Pers. Soc. Psychol. 54, 1063-1070. doi:10.1037/00223514.54.6.1063

Zubieta, J.K., Ketter, T.A., Bueller, J.A., Xu, Y.J., Kilbourn, M.R., Young, E.A., Koeppe, R.A., 2003. Regulation of human affective responses by anterior cingulate and limbic mu-opioid neurotransmission. Arch. Gen. Psychiatry 60, 1145-1153. 


\section{Figure captions}

\section{Figure 1.}

An illustration of the Stroop-like task that we employed. In each trial, participants indicated the direction specified by a target word (left, right, up, or down) that followed a 3-word distracter (left panel). The 3word distracter was mapped to the same response as the target (congruent trials) or to a different response (incongruent trials). To avoid feature integration confounds, we constructed the task so that participants would alternate between trials involving a left-right task and an up-down task. Critically, this meant there were no stimulus or response repetitions in consecutive trials. We conducted sequential analyses to investigate the influence of performance errors and incongruent trials in trial $\mathrm{N}$ on performance adaptations in the subsequent trial (trial $\mathrm{N}+1$ ). We measured two aspects of adaptive control (see Methods): (1) post-error slowing (middle panel) and (2) conflict adaptation (right panel).

\section{Figure 2.}

Relative to placebo, naltrexone boosted adaptive control following errors, as indexed by greater post-error slowing, $p=.002$. The figure plots mean \pm standard error. Each circle indicates post-error slowing (in ms) for an individual participant. 
Table 1.

\begin{tabular}{|c|c|c|c|c|c|c|}
\hline \multirow[t]{2}{*}{ Measure } & \multicolumn{3}{|c|}{ Placebo $(\mathrm{N}=22)$} & \multicolumn{3}{|c|}{ Naltrexone $(\mathrm{N}=18)$} \\
\hline & Mean & SE & $95 \% \mathrm{Cl}$ & Mean & SE & $95 \% \mathrm{Cl}$ \\
\hline RT pre-error (ms) & 531 & 34 & {$[461,602]$} & 452 & 29 & {$[391,513]$} \\
\hline RT post-error (ms) & 548 & 28 & {$[490,607]$} & 584 & 28 & {$[524,644]$} \\
\hline Post Error Slowing (ms) & 17 & 27 & {$[-39,74]$} & 133 & 27 & {$[76,189]$} \\
\hline Accuracy post-correct (\%) & 95.4 & 0.6 & {$[96.6,94.1]$} & 95.3 & 0.8 & {$[97.0,93.5]$} \\
\hline Accuracy post-error (\%) & 98.5 & 0.9 & {$[100.3,96.7]$} & 98.3 & 0.8 & {$[100.0,96.6]$} \\
\hline Post Error Accuracy (\%) & 3.2 & 0.8 & {$[1.6,4.7]$} & 3.1 & 0.9 & {$[1.2,5.0]$} \\
\hline $\mathrm{RT} c \mathrm{c}(\mathrm{ms})$ & 460 & 22 & {$[413,507]$} & 437 & 21 & {$[394,481]$} \\
\hline $\mathrm{RT} c \mathrm{l}(\mathrm{ms})$ & 563 & 25 & {$[512,615]$} & 555 & 24 & {$[504,607]$} \\
\hline $\mathrm{RT}$ iC (ms) & 472 & 22 & {$[427,518]$} & 453 & 18 & {$[415,491]$} \\
\hline RT il (ms) & 553 & 24 & {$[503,604]$} & 538 & 21 & {$[495,582]$} \\
\hline RT conflict-adaptation effect (ms) & 22 & 9 & {$[3,41]$} & 32 & 10 & {$[10,54]$} \\
\hline RT interference effect (ms) & 92 & 9 & {$[74,110]$} & 102 & 11 & {$[78,126]$} \\
\hline RT grand average $(\mathrm{ms})$ & 512 & 23 & {$[465,559]$} & 496 & 20 & {$[454,538]$} \\
\hline Accuracy cC (\%) & 95.3 & 0.9 & {$[97.2,93.4]$} & 95.1 & 1.1 & {$[97.5,92.8]$} \\
\hline Accuracy cl (\%) & 94.1 & 0.8 & {$[95.8,92.4]$} & 92.9 & 1.0 & {$[95.0,90.8]$} \\
\hline Accuracy iC (\%) & 97.7 & 0.5 & {$[98.7,96.7]$} & 97.4 & 0.8 & {$[99.1,95.8]$} \\
\hline Accuracy il (\%) & 94.1 & 1.2 & {$[96.5,91.7]$} & 95.4 & 0.9 & {$[97.3,93.6]$} \\
\hline Accuracy conflict-adaptation effect (\%) & -2.4 & 1.6 & {$[-5.7,1.0]$} & 0.2 & 0.8 & {$[-1.5,2.0]$} \\
\hline Accuracy interference effect (\%) & 2.4 & 0.6 & {$[1.2,3.6]$} & 2.1 & 0.6 & {$[0.8,3.4]$} \\
\hline Accuracy grand average (\%) & 95.2 & 0.6 & {$[96.5,94.0]$} & 95.2 & 0.8 & {$[96.9,93.5]$} \\
\hline Positive Affect & 2.8 & 0.1 & {$[2.5,3.1]$} & 2.7 & 0.2 & {$[2.4,3.1]$} \\
\hline Negative Affect & 1.4 & 0.1 & {$[1.2,1.6]$} & 1.4 & 0.1 & {$[1.2,1.6]$} \\
\hline
\end{tabular}




\section{Figures}

\section{FIGURE 1}

Trial sequence:

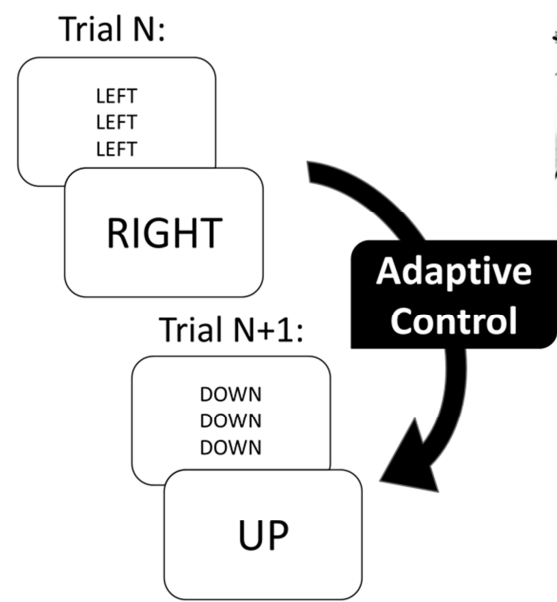

1. Post-error adaptation:

2. Conflict adaptation:

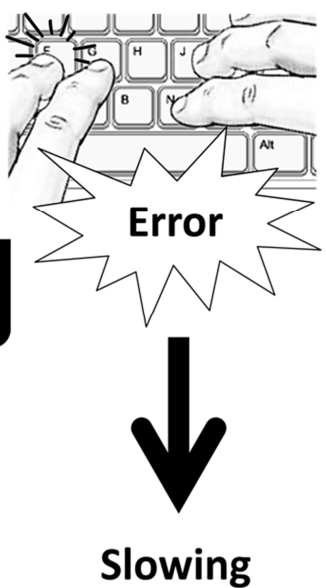

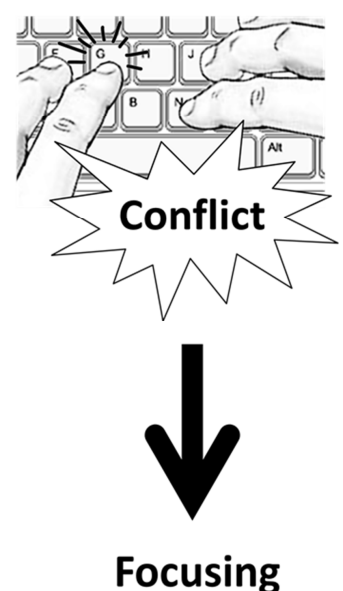

Figure 1. An illustration of the Stroop-like task that we employed. In each trial, participants indicated the direction specified by a target word (left, right, up, or down) that followed a 3-word distracter (left panel). The 3-word distracter was mapped to the same response as the target (congruent trials) or to a different response (incongruent trials). To avoid feature integration confounds, we constructed the task so that participants would alternate between trials involving a left-right task and an up-down task. Critically, this meant there were no stimulus or response repetitions in consecutive trials. We conducted sequential analyses to investigate the influence of performance errors and incongruent trials in trial $\mathrm{N}$ on performance adaptations in the subsequent trial (trial $\mathrm{N}+1)$. We measured two aspects of adaptive control (see Methods): (1) post-error slowing (middle panel) and (2) conflict adaptation (right panel) 
FIGURE 2

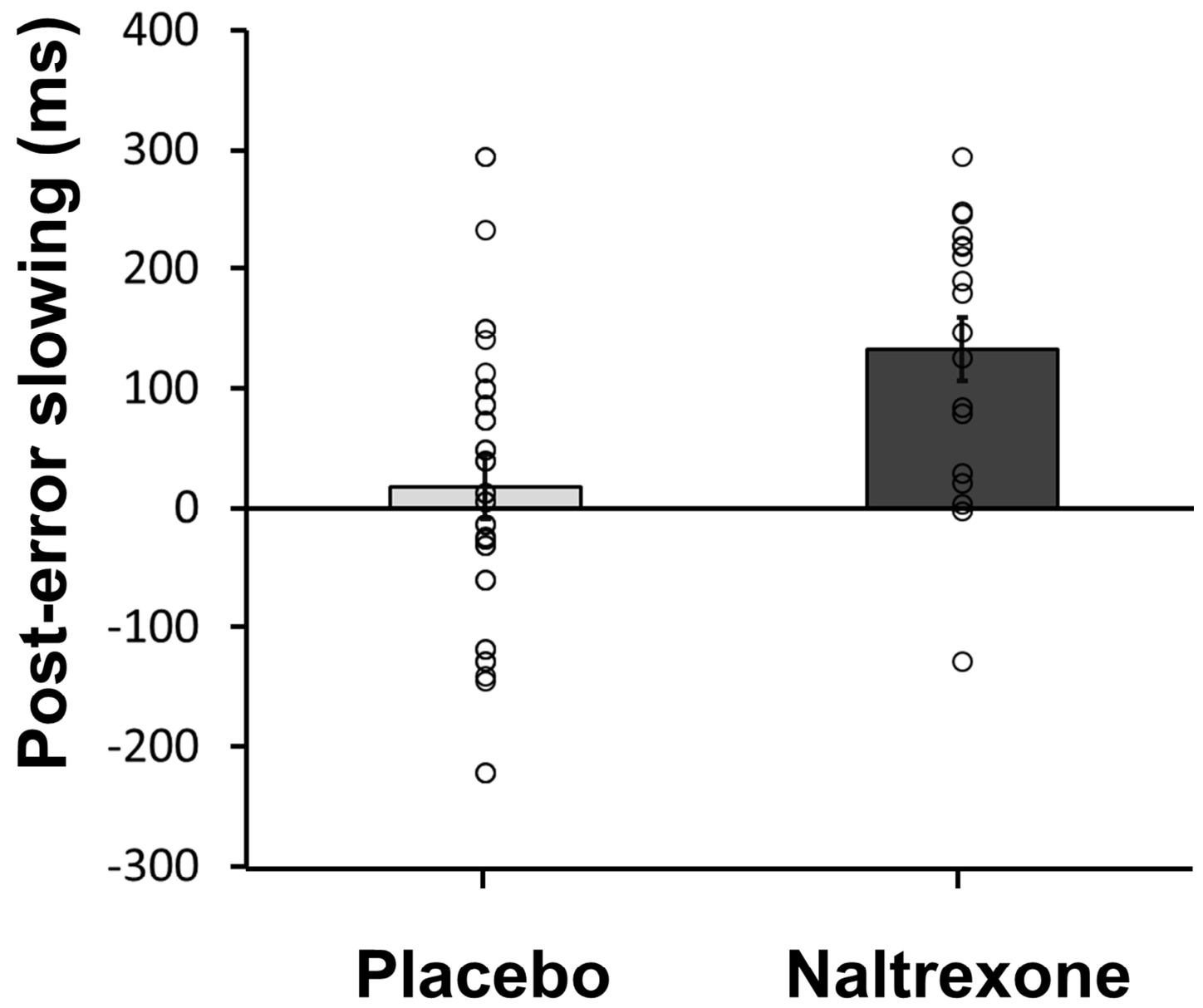

Figure 2. Relative to placebo, naltrexone boosted adaptive control following errors, as indexed by greater post-error slowing, $p=.005$. The figure plots mean \pm standard error. Each circle indicates post-error slowing (in $\mathrm{ms}$ ) for an individual participant. 